\title{
Thermal conductivity Characterization of Bamboo fiber reinforced in Epoxy Resin
}

\author{
E.chandana ${ }^{1 *}$, Dr.Syed Altaf Hussian ${ }^{2}$, \\ ${ }^{1 *} P G$ student, RGM college of Engineering \&Technology, Nandyal-518501, INDIA \\ ${ }^{2}$ Department of Mechanical Engineering, RGM college of Engineering \&Technology, Nandyal- 518501, INDIA
}

\begin{abstract}
Over a past few decades composites, plastics, ceramics have been the dominant engineering material. The areas of applications of composites materials have grown rapidly and have even found new markets. The current challenge is to make the durable in tough conditions to replace other materials and also to make them cost effective. This has resulted in development of many new techniques currently being used in the industry. While the use of composites it is clear choice in many applications but the selection of material will depend on the factor such as working life, lifetime requirement, complexity of product shape produced, saving the term cost. The availability of natural fiber is abundances and also they are very inexpensive when compared to other advanced manmade fibers. The primary advantage of natural fibers are low density, low cost, biodegradability, acceptable specific properties, less wear during extracting as well as manufacturing composites and wide varieties of natural fibers are locally available. The main focus of this investigation is to determine the thermal conductivity of bamboo fiber reinforced in epoxy resin composites. The test samples were prepared as per ASTM standards using simple hand-layup technique at different fiber weight fractions $(10 \%$, $20 \% 30 \%, 40 \% 50 \%, 60 \%)$. Thermal conductivity $(\mathrm{K})$ of the composites material were determined experimentally and is validated by the results obtained by rule of mixture, E-S model and also by finite element modeling
\end{abstract}

Keyword: Bamboo fiber, Epoxy, Thermal conductivity, guarded heat flow.

\section{Introduction}

The word composite in the term composite material signifies that two or more materials are combined on a macroscopic scale to form a useful third material. The key is the macroscopic examination of a material where in the components can be identified by the naked eye. Different materials can be combined on a microscopic scale, such as in alloying of metals, but the resulting material is, for all practical purposes macroscopically homogeneous, i.e., the components cannot be distinguished by the naked eye and essentially act together. The advantage of composite material is that, if will designed, they usually exhibit the best qualities of their components or constituents and often some qualities that neither constituent possesses. The advantage of composite materials over conventional materials are largely from their higher specific strength, stiffness and fatigue characteristics, which enables structural design to be more versatile. By definition, composite materials consist of two or more constituents with physically separable phases. However, only when the composite phase materials have notably different physical properties it is recognized as being a composite material. Reinforcement provides strength and rigidity, helping to support structural load. The matrix or binder (organic or inorganic) maintains the position and orientation of the reinforcement. Thermoplastics occupy only a small percentage of the advanced composite market, while other and epoxy thermoset materials contribute to more than 70 per cent. Epoxy resins the most important matrix polymer when it comes to high performance. Natural fibers such as bamboo, coir, jute, flux, sisal etc., are low density fibers available at low cost. Natural fibers are renewable and also easily biodegradable. In spite of having several merits, natural fibers show lower modulus, lower strength and poor resistance when compared with the composites reinforced with synthetic fibers such as glass, carbon and aramid. To overcome these limitations and to obtain great diversity of material Composites Materials are combinations of two phases in which one of the phases, called the reinforcing phase, which is in the form of fiber sheets or particles and are embedded in the other phase called the matrix phase. The primary functions of the matrix are to transfer stresses between the reinforcing fibers or particles and to protect them from mechanical and environmental damage whereas the presence of fibers or particles in a composite improves its mechanical properties such as strength, stiffness etc. A composite is therefore a synergistic combination of two or more micro-constituents which differ in physical form and chemical composition and which are insoluble in each other. Our objective is to take advantage of the superior properties of both materials without compromising on the weakness of either. Composite materials have successfully substituted the conventional materials in several applications like light weight and high strength. The reasons why composites are selected for such applications are mainly due to their high strength-to-weight ratio, high tensile strength at elevated temperatures, high creep resistance and high toughness. Typically, the reinforcing materials are strong with low densities while the matrix is usually a ductile or tough material. If the composite is designed and fabricated 
correctly it combines the strength of the reinforcement with the toughness of the matrix to achieve a combination of desirable properties not available in any single traditional material. The strength of the composites depends primarily on the amount, arrangement and type of fiber or particle reinforcement in the resin. Fiber reinforced polymers offer advantages over other conventional materials when specific properties are compared. That's the reason for these composites finding applications in diverse fields from appliances to spacecrafts. Natural fibers are largely divided into two categories depending on their origin: plant based and animal based. Therefore, natural fiber can serve as reinforcements by improving the strength and stiffness and also reducing the weight of resulting biocomposites materials, although the properties of natural fibers vary with their source and treatments.

Fadhel Abbas Abdullah et al. [1] Investigated on natural composites of jute and white feature as there raw material for finding thermal conductivity and concluded that increasing in volume in volume fraction of natural fiber causes decreases in thermal conductivity.

Monika et al. [2] focused on Thermal conductivity of bamboo fiber reinforced composite by varying volume fraction, fiber length concluded increase in volume fraction of fiber by decreases in thermal conductivity.

\subsection{Material}

\section{Material And Methods}

High performance epoxy resin LY 556 and the curing agent hardener HY 951 system were used as the matrix. Bamboo fibers were procured from Tripura state of India in the dried form. Some of these fibers were soaked in 3moles of $\mathrm{NaOH}$ solution for $30 \mathrm{~min}$. to remove any greasy material and hemi cellulose, washed thoroughly in distilled water and dried under the sun for one week.

\subsection{Composite Manufacturing}

Composites are prepared as per the ASTM E-1530 standards. The foremost required resin mixture is prepared by adding accelerator and catalyst $10 \%$ to resin at room temperature. The samples were prepared using Hand -lay up technique was adopted to fill up the prepared mould with an appropriate amount of polymer resin mixture and bamboo fiber. For making the composite, a molding box was prepared with leather as per the ASTM standards with $200 \mathrm{~mm}$ X $100 \mathrm{~mm} \mathrm{X} 3 \mathrm{~mm}$ mould cavity. Fiber and epoxy resin is mixed slowly and filled in mold without any bubbles. Therfore at the time of curing a some compression pressure is applied on the mold and the composite specimen were cured for $24 \mathrm{hrs}$.the specimen were also post cured at $70^{\circ} \mathrm{c}$ for $2 \mathrm{hrs}$ after removing from the mold.

\subsection{Preparation of Test Specimens}

Thermal conductivity testing samples were prepared in cylindrical shape as per ASTM E-1503 $(50 \mathrm{~mm}$ diameter and $3 \mathrm{~mm}$ thickness) by changing the fiber loading fraction $(10 \%, 20 \%, 30 \%, 40 \%, 50 \%$ and $60 \%)$.

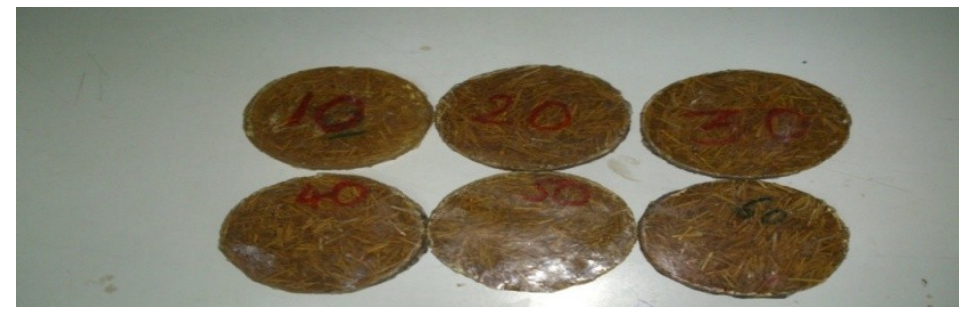

Figure1: samples pieces for testing Thermal conductivity

\subsection{Experimental test for Thermal conductivity}

The specimens were tested by using guarded heat flow meter (Unitherm model 2022, ANTER Corp., Pittsburgh, PA).the following equation(1) is used for calculation of thermal conductivity

$$
\begin{aligned}
& \mathrm{Q}=\frac{\mathrm{KA}(\mathrm{t} 1-\mathrm{t} 2)}{\mathrm{x}} \\
& \mathrm{R}=\frac{\mathrm{t} 1-\mathrm{t} 2}{\mathrm{q}} \\
& \mathrm{K}=\frac{\mathrm{x}}{\mathrm{R}}
\end{aligned}
$$

Where $\mathrm{Q}$ is the heat flux $(\mathrm{W}), \mathrm{K}$ is the thermal conductivity $(\mathrm{W} / \mathrm{m}-\mathrm{K}), \mathrm{A}$ is the cross- sectional area $(\mathrm{m} 2), \mathrm{t} 1-\mathrm{t} 2$ is the difference in temperature $(\mathrm{K})$ and $\mathrm{x}$ is the thickness of the sample, $\mathrm{R}$ is the resistance of the sample between hot and cold surfaces $(\mathrm{m} 2-\mathrm{K} / \mathrm{W})$. 


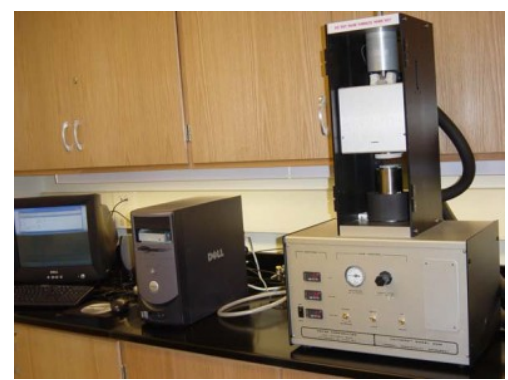

Fig: 2 Experimental set up of Unitherm 2022

In Unitherm 2022 the heat flux transducer measures the $\mathrm{Q}$ value and the temperature difference can be obtained between the upper plate and lower plate. Thus the thermal resistance can be calculated between the upper and lower surfaces. Giving the input value of thickness and taking the known.

\subsection{Theoretical test for thermal conductivity}

To date, several models for determining the thermal conductivity of polymer composites have been proposed. There are many types of composite systems in which a polymer is reinforced with unidirectional arranged fibers. In those systems, the thermal conductivity of the composite is the highest. This is due to the anisotropic nature of fibers; while the conductivity is good along the fiber, the heat flow across the fiber is poor. On the other hand, if the alignment of fibers is perpendicular to the direction of thermal flux, the thermal conductivity will be the lowest. The thermal conductivity of fiber and matrix have been evaluated by extrapolating the linear regression of thermal conductivity values of the composite to $100 \%$ fiber and $0 \%$ fiber and are found be $0.07 \mathrm{~W} / \mathrm{mK}$ and $0.368 \mathrm{~W} / \mathrm{mK}$ respectively. The behavior of the thermal conductivity of different composites can now be explained using thermal conductivity values of the fiber. Further, the measured thermal conductivity of composites was compared with series model (Rule of mixture) and E-S model. The expressions for these two

\subsubsection{Rule of mixture or Series model:}

There are many types of composite systems in which a polymer is reinforced with fibres. In those systems, thermal conductivity of composite is the highest. This is because the fibres are gathered in the polymer to form a conductive block of polymer whose alignment of conductive block of polymer is perpendicular to the direction of thermal flux the thermal conductivity will be the lowest (series model).

$$
\frac{1}{\mathrm{Kc}}=\frac{\mathrm{Vf}}{\mathrm{Kf}}+\frac{1-\mathrm{Vf}}{\mathrm{Km}}
$$

Where $\mathrm{Vf}$ and $\mathrm{Kf}$ denote the volume fraction and the thermal conductivity of fiber, respectively, while $\mathrm{km}$ is the thermal conductivity of matrix.

\subsubsection{E-S model}

A general expression for effective transverse thermal conductivities of unidirectional composites was derived by Zou et al.The model was presented based on the thermal- electrical analogy technique for elliptical filament and square packing array unit cell model (E-S model). The dimensionless effective transverse thermal conductivity, $k$ e, the ratio of the thermal conductivity of the composite, $k$ c, to the thermal conductivity of matrix, $\mathrm{km}$, were expressed as a function of the ratio of thermal conductivities of filler to matrix, $\beta$, filler volume fraction, Vf, and the geometry ratio of the filler, $\rho=a / b$, where $\mathrm{a}$ and $\mathrm{b}$ are the axial lengths of the ellipse along the $x$-axis and $y$-axis, respectively

$$
\begin{aligned}
& \mathrm{Ke}=\frac{\mathrm{Kc}}{\mathrm{km}}=1-\frac{1}{\mathrm{c}}+\frac{\pi}{2 \mathrm{~d}}-\frac{\mathrm{c}}{\sqrt[\mathrm{d}]{\mathrm{d}^{2}-\mathrm{c}^{2}}} \ln \left|\mathrm{d}+\sqrt{\frac{\mathrm{d}^{2}-\mathrm{c}^{2}}{\mathrm{c}}}\right| . \\
& \mathrm{C}=\sqrt{\frac{\frac{\pi \rho}{\mathrm{Vf}}}{2}}, \mathrm{~d}=\rho\left(\frac{1}{\beta}-1\right), \beta=\frac{\mathrm{Kf}}{\mathrm{Km}}
\end{aligned}
$$

When $\rho=1$ (i.e., $a=b$ ), the present model can be simplified as the cylindrical filaments in a square packing array unit cell model.

\subsection{Experimental test for Thermal conductivity}

\section{Result And Disscusion}

In this work composite material is fabricated using hand layup method. The experiment were conducted by using guarded heat flow meter (Unitherm model 2022, ANTER Corp., Pittsburgh, PA )and Table 1 shows the result of thermal conductivity for various weight fraction of fiber 


\begin{tabular}{|c|c|c|}
\hline s.no & Fiber weight fraction(Wf)\% & Thermal conductivity(w/m-k) \\
\hline 1 & 10 & 0.197 \\
\hline 2 & 20 & 0.195 \\
\hline 3 & 30 & 0.1889 \\
\hline 4 & 40 & 0.1880 \\
\hline 5 & 50 & 0.184 \\
\hline 6 & 60 & 0.173 \\
\hline
\end{tabular}

Table1: Variation of thermal conductivities for varying weight fraction of fiber

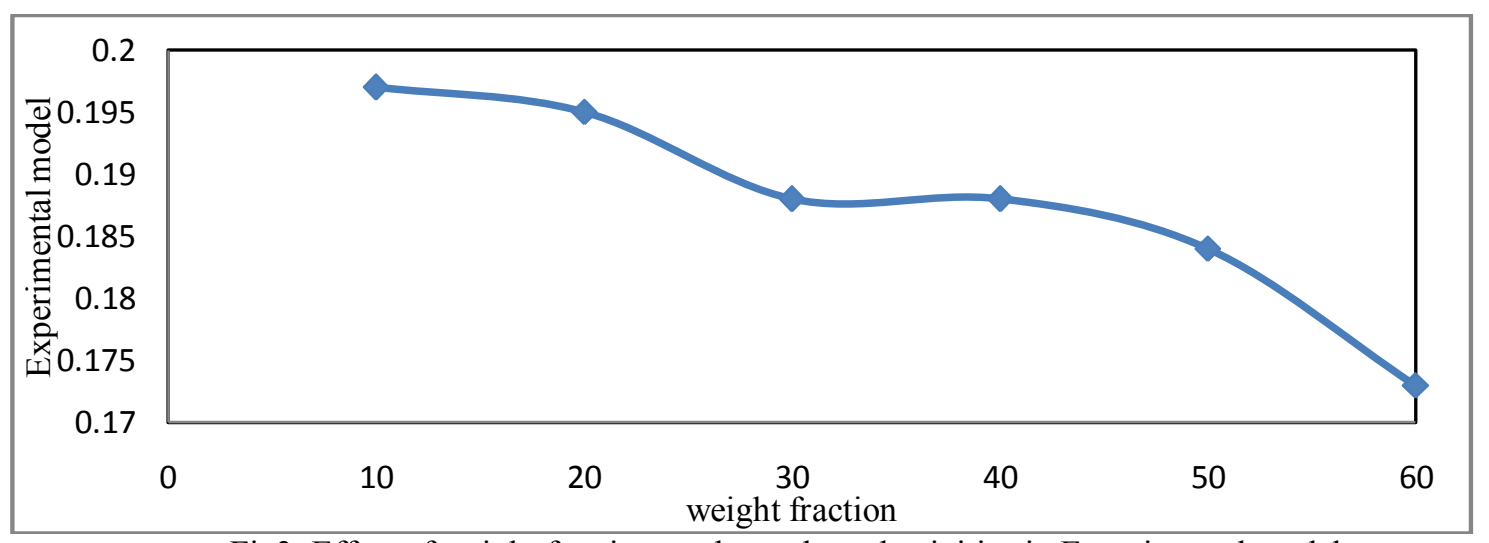

Fig3: Effect of weight fraction on thermal conductivities in Experimental model

\subsection{Theoretical test for thermal conductivity}

The calculated thermal conductivity of bamboo fiber polymer composites as a function of fiber content are presented in (fig 2) and (fig 3).It is observed that the theoretical model overestimated the values of thermal conductivity with respect to the experimental model. This may be attributed to the fact that some of the assumptions taken to the model are not practical. Further, in two theoretical models, orientation of the fibers was assumed to be perfect, but in actual practice when liquid matrix is poured over fibers some of the fibers may be misaligned to each other and table 2 shows the result series model of thermal conductivity by varying weight fraction of fiber and table 3 shows the result E-s model of thermal conductivity by varying weight fraction.

Table2: Variation of thermal conductivities on varying weight fraction

\begin{tabular}{|c|c|c|}
\hline S .no & Fiber weight fraction(Wf)\% & Thermal conductivity (w/m-k) \\
\cline { 3 - 3 } & & Series model \\
\hline 1 & 10 & 0.216 \\
\hline 2 & 20 & 0.168 \\
\hline 3 & 30 & 0.144 \\
\hline 4 & 40 & 0.130 \\
\hline 5 & 50 & 0.120 \\
\hline 6 & 60 & 0.113 \\
\hline
\end{tabular}

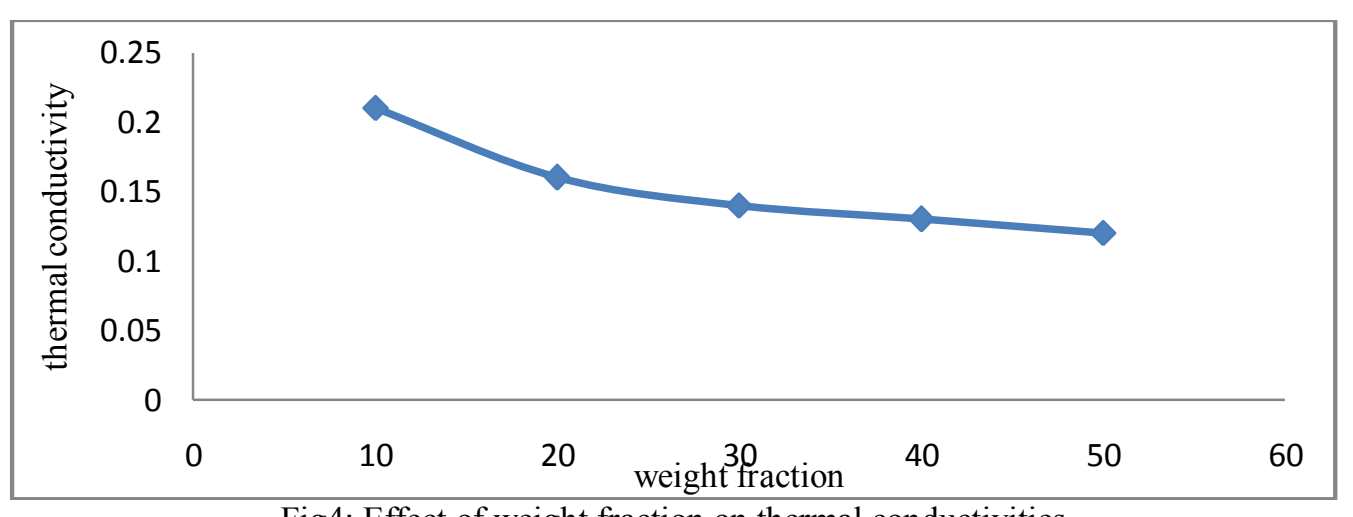

Fig4: Effect of weight fraction on thermal conductivities 
Table3: Variation of thermal conductivities for varying weight fraction

\begin{tabular}{|c|c|c|}
\hline s.no & Fiber weight fraction(Wf)\% & Thermal conductivity (w/m-k) \\
\cline { 3 - 3 } & & E-S model \\
\hline 1 & 10 & 0.259 \\
\hline 2 & 20 & 0.257 \\
\hline 3 & 30 & 0.126 \\
\hline 4 & 40 & 0.120 \\
\hline 5 & 50 & 0.116 \\
\hline 6 & 60 & 0.111 \\
\hline
\end{tabular}

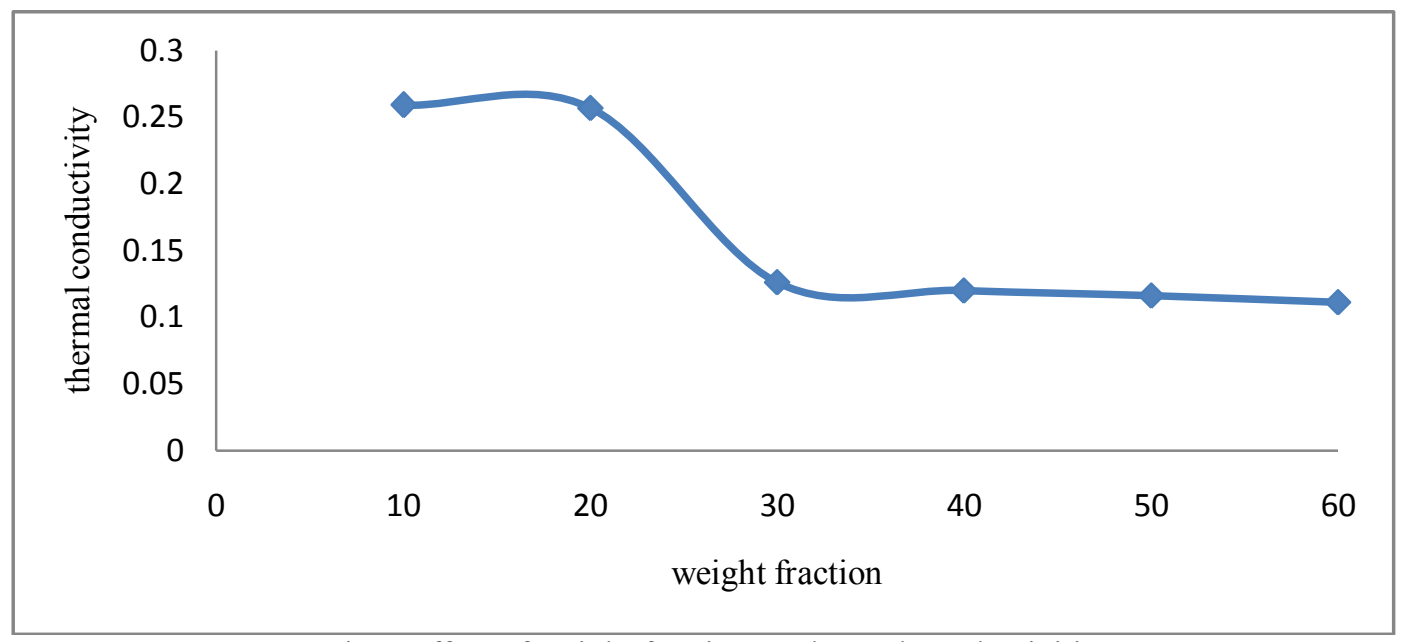

Fig5: Effect of weight fraction on thermal conductivities

\subsection{Thermal Analysis by using Ansys software}

In this study, Ansys software has been used in the finite element analysis to conduct the thermal analysis of composite structure. The Finite Element Method (FEM), originally introduced by Turner in 1956, is a powerful computational technique for approximate solutions to a variety of "real-world" engineering problems having complex domains subjected to general boundary conditions. FEM has become an essential step in the design or modelling of a physical phenomenon in various engineering disciplines. A physical phenomenon usually occurs in a continuum of matter (solid, liquid, or gas) involving several field variables. The field variables vary from point to point, thus possessing an infinite number of solutions in the domain. The FEM is thus a numerical procedure that can be used to obtain solutions to a large class of engineering problems involving stress analysis, heat transfer, fluid flow etc. ANSYS is general-purpose finite-element modelling package for numerically solving a wide variety of mechanical problems that include static/dynamic, structural analysis (both linear and nonlinear), heat transfer, and fluid problems, as well as acoustic and electromagnetic problems. The main objective to applying thermal analysis is to figure out the temperatures and heat flow on each node generated by ANSYS for numerical solution in composite structure.

\subsubsection{Model Development}

Bamboo fiber reinforced in epoxy resin has investigated in two-dimensional. Two-dimensional model have been developed for fiber-reinforced composites. For 2-D model, shape of the matrix material has been chosen as infinitely long square prism; whereas shape of the fiber have been chosen as infinitely long cylinder. The dimensions of the square of the matrix is fixed unity and the fiber dimension is varying as $\varphi(20 \%)$.In the numerical analysis of the heat conduction problem, the temperatures at the nodes along the surfaces is prescribed as T1 (303k) ambient temperature and another side T2 (333k). The other surfaces parallel to the direction of the heat flow are all assumed adiabatic. The temperatures at the nodes in the interior region and on the adiabatic boundaries are unknown. These temperatures are obtained with the help of finite-element program package ANSYS.

Formula for finding thermal conductivity:

Where $\mathrm{q}=$ Heat flux

$$
\begin{array}{r}
K=\frac{q}{A} \\
A=\frac{d t}{d x}
\end{array}
$$


$\mathrm{A}=$ Thermal gradient

$\mathrm{dt}=$ temperature difference between surfaces

Meshing model:
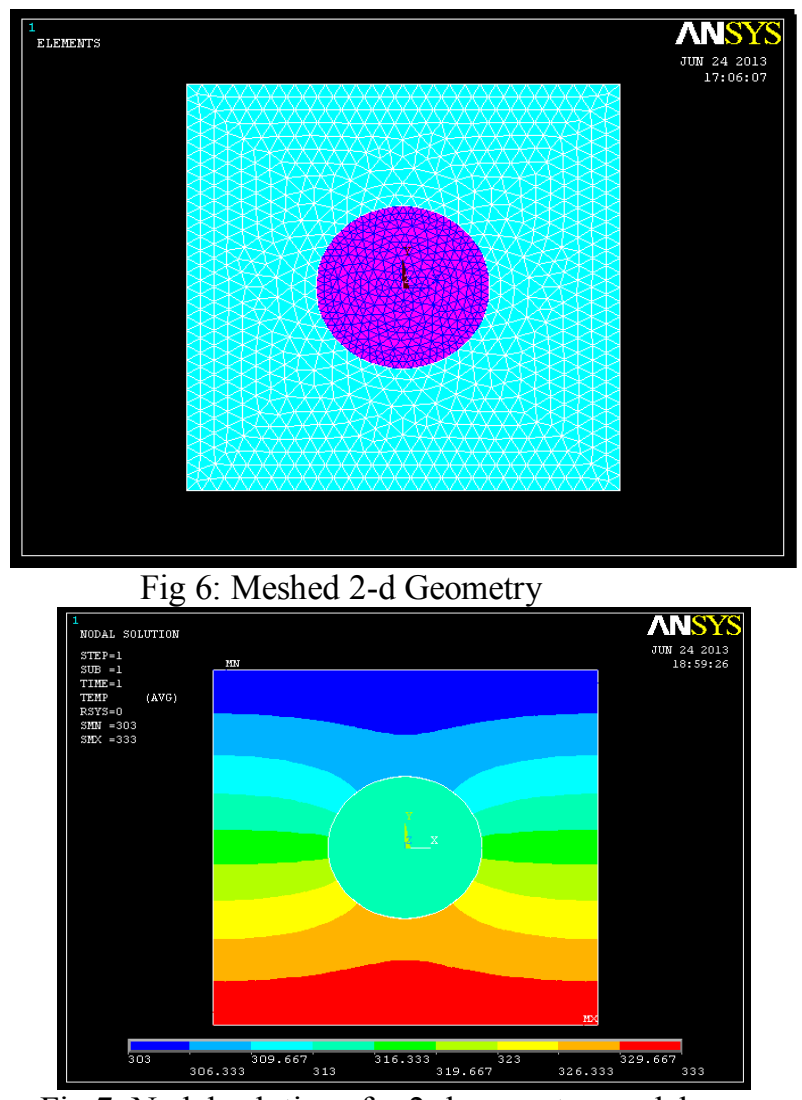

Fig 7: Nodal solutions for 2-d geometry model

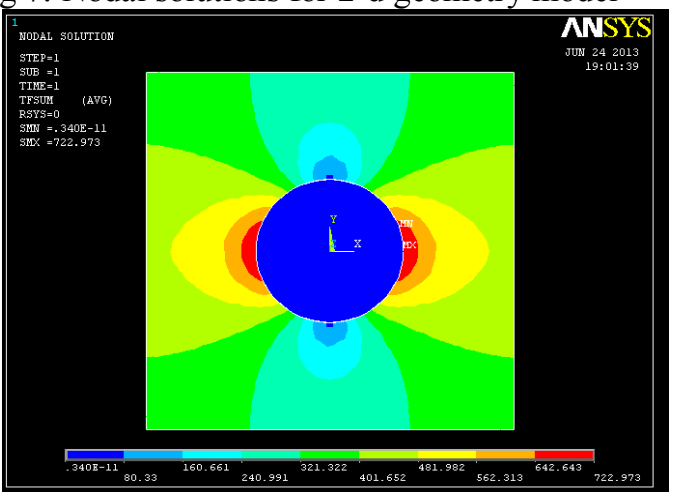

Fig 8: Heat Flux for 2-d geometry model.

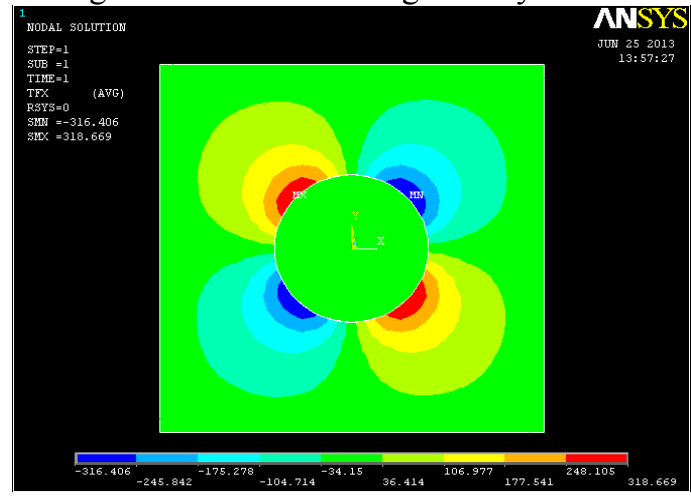

Fig: 9 Thermal Flux for 2-d geometry model 


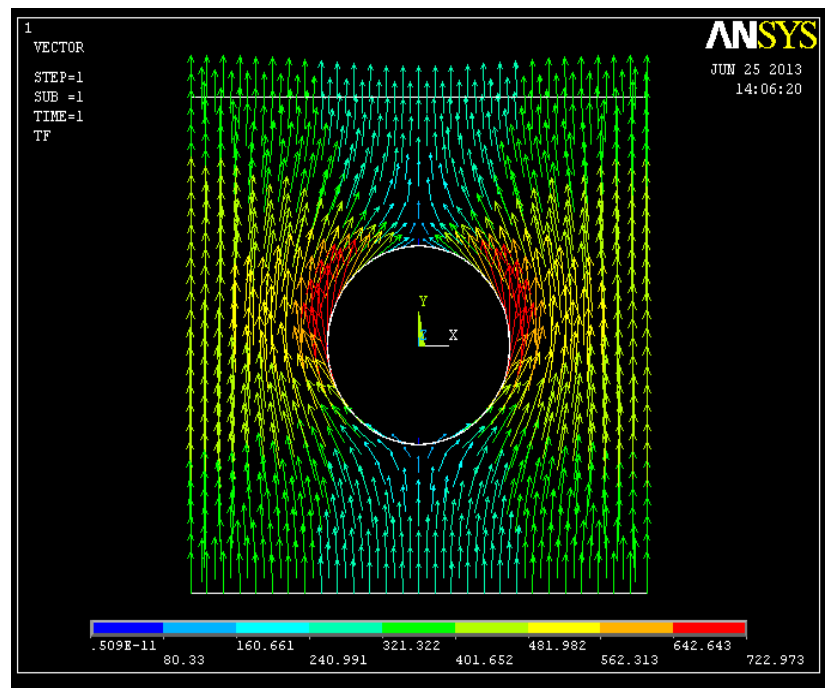

Fig: 10 Vector Sum for 2-d geometry model

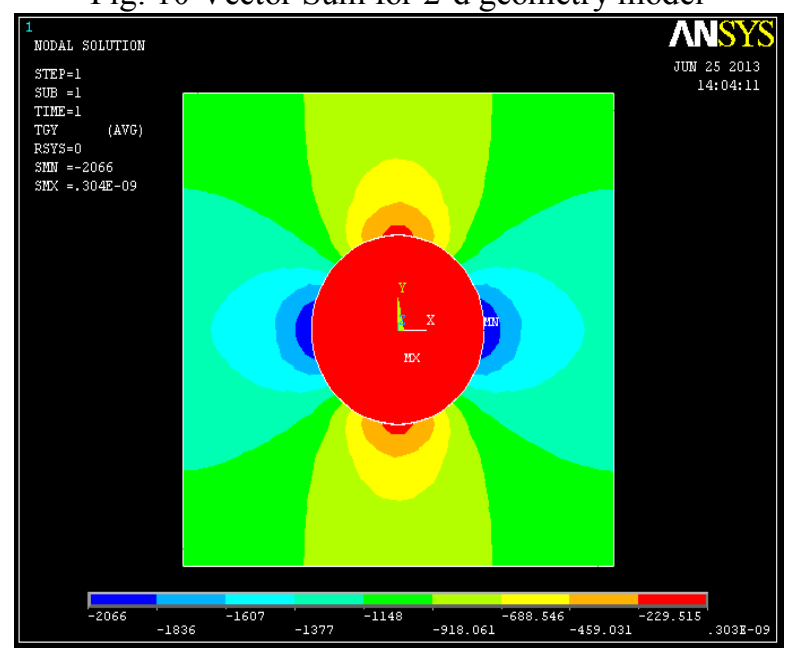

Fig: 11 Heat Gradient for 2-d geometry model

\begin{tabular}{|c|c|c|c|}
\hline sample & $\begin{array}{l}\Phi(\%) \\
\text { weight \% of fiber }\end{array}$ & $\begin{array}{l}\text { Experimental } \\
\text { values(k)w/m-k }\end{array}$ & Ansys value(k)w/m-k \\
\hline 1 & 20 & 0.195 & 0.26 \\
\hline 2 & 30 & 0.188 & 0.23 \\
\hline 3 & 50 & 0.184 & 0.20 \\
\hline
\end{tabular}

Table4: Thermal conductivity between ansys and experimental values

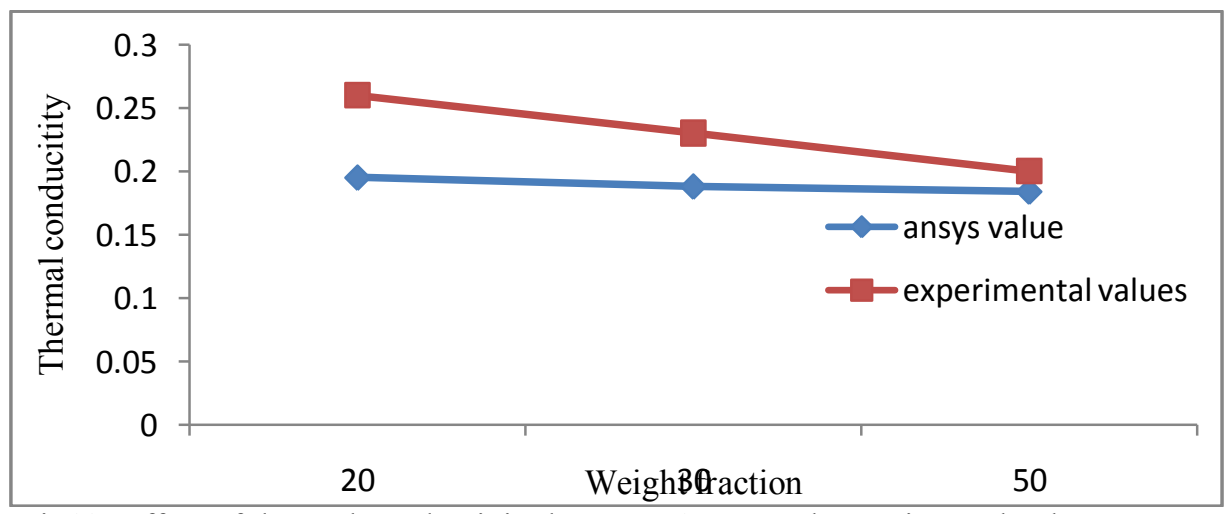

Fig13: Effect of thermal conductivity between Ansys and experimental values 


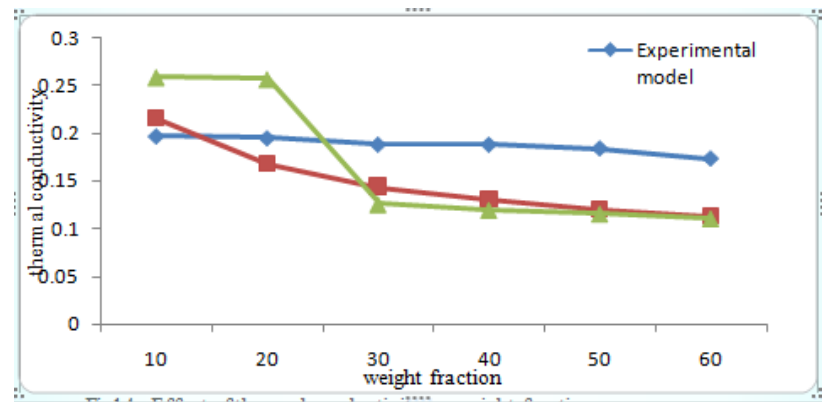

Fig14: Effect of thermal conductivity on weight fraction

\section{Conculsion}

In this investigation, partially biodegradable green composites with various weight fractions of bamboo fibers were successfully developed at various fiber weight fractions and their thermal conductivity $(\mathrm{k})$ were determined experimentally and is validated by the result obtained by, theoretically and also using Finite element Analysis.

- The thermal conductivity of the composites has decreased with increases in weight fraction of fiber in experimentally, theoretically and also in finite element analysis.

- At 10\%weight fraction of fiber, it was noticed that all the models (Experimental, rule of mixture model E-s model) were showing high thermal conductivity.

- The thermal conductivity experimental result values are as near to rule of mixture model. The \%error between the experimental model and rule of mixture is less when compare to the E-s model.

- At $10 \%$ weight fraction of fiber only $0.9 \%$ of error in rule of mixture model.

- Bamboo fiber reinforced composite is lowest value of thermal conductivity among glass fiber reinforced composite, coconut fiber, carbon fiber

- The result of this investigation indicates that the bamboo fibers composite is light in weight, economical and possess good thermal insulating. Hence, the newly developed composite material can be used for the application such as automobile like body panels, corrosion of sensitive parts, drive train ,suspension system, electronic packages, aerospace like blades, interiors design, air frames, building construction like rebar, seismic retrofits and sports goods.

- Hence from this investigation we can conclude that experimental result values are as nearer to the theoretical model and finite element analysis.

\section{Acknowledgement}

It is my privilege to express my thanks to my guide Dr. SYED ALTAF HUSSAIN, M.Tech, Ph.D., Professor in Mechanical Engineering, R.G.M College of Engineering and Technology, for his valuable guidance and encouragement.

[1] Syed Altaf Hussian, V. Pandurangadu, .Mechanical properties of green coconut fiber reinforced HDPE polymer composite.3(11),2011,9075-5462.

[2] Syed Altaf Hussian, V. Pandurangadu, Mechanical properties of short bamboo fiber reinforced polyester composites filled with alumina particulate,2(3),2012,2250-3498.

[3] M.Mounika, k.Ramaniah .Thermal conductivity characterization of bamboo fiber reinforced polyester composite.3 (6)2012, 11091116.

[4] Dr. Fadhel Abbas Abdullah, Theoretical and Experimental Investigation of Natural Composite Materials as Thermal Insulation, 4 (2), 2011.

[5] Symington Mc, BanksWM, WestOpukuro Dravid, Pethrick, Journal of CompositeMaterials, 4(3)2009, 1083

[6] Jonathan D.Mar, Efim Litovsky and Jacobs Kleiman, Journal of building physics, 32(2008)9.

[7] Yamashita yoshihiro, Yamada hiroaki, Thermal conductivity of plain weave fabric and its composite material made from high strength fibers.

[8] Sreenivasan VS, Soman Sundram S, Naryanaswamy R, Material and Design, 32(2011)453.

[9] RavindraMangal,N.S.Saxena,M.s.Sreekala,S.Thomas,CompositesScienceandTechnology,66(2006),2719-2725.

[10] Serhat Durmaz,.Numerical study on the effective thermal conductivity of composite materials.

[11] Bhyrav Mutnuri, Aksogan Orhan, Thermal conductivity characterization of composite material. ANTEC 2006, May 7-11.

[12] Murali Mohan Rao K, Ratna Prasad AV, Material \&design, 3(2010)508.

[13] E. Trujillo, L. Osorio (2010), Characterization of Polymer Composite Materials Based On Bamboo Fibres.

[14] Yibin Xu, Junichi Kinugawa and Koichi Yagi (2003). Development of Thermal Conductivity Prediction System for Composites.

[15] Ali M. Othman (2010), Experimental Investigations of the Effect of Some Insulating Materials on the Compressive Strength, Water Absorption and Thermal Conductivity of Building Bricks.

[16] Kalaprasad G, Pradeep P, Mathew G, Composite Science \&Technology, 60(2000) 2967. 